\title{
Fluid lipid bilayers: Intermonolayer coupling and its thermodynamic manifestations
}

\author{
Hansen, Per Lyngs; Miao, Ling; Ipsen, John Hjorth
}

Published in:

Physical Review E. Statistical, Nonlinear, and Soft Matter Physics

Link to article, DOI:

10.1103/PhysRevE.58.2311

Publication date:

1998

Document Version

Publisher's PDF, also known as Version of record

Link back to DTU Orbit

Citation (APA):

Hansen, P. L., Miao, L., \& Ipsen, J. H. (1998). Fluid lipid bilayers: Intermonolayer coupling and its

thermodynamic manifestations. Physical Review E. Statistical, Nonlinear, and Soft Matter Physics, 58(2), 2311. 2324. https://doi.org/10.1103/PhysRevE.58.2311

\section{General rights}

Copyright and moral rights for the publications made accessible in the public portal are retained by the authors and/or other copyright owners and it is a condition of accessing publications that users recognise and abide by the legal requirements associated with these rights.

- Users may download and print one copy of any publication from the public portal for the purpose of private study or research.

- You may not further distribute the material or use it for any profit-making activity or commercial gain

- You may freely distribute the URL identifying the publication in the public portal 


\title{
Fluid lipid bilayers: Intermonolayer coupling and its thermodynamic manifestations
}

\author{
Per Lyngs Hansen,* Ling Miao, and John Hjort Ipsen \\ Department of Chemistry, Building 207, The Technical University of Denmark, DK-2800 Lyngby, Denmark
}

(Received 8 December 1997)

\begin{abstract}
A fluid membrane of lipid bilayer consists of two individual molecular monolayers physically opposed to each other. This unique molecular architecture naturally necessitates the need to treat a lipid-bilayer membrane as one entity of two coupled two-dimensional systems (monolayers), each of which possesses "in-plane", degrees of freedom that characterize its physical or chemical state. Thermally excitable deformations of a lipid bilayer in its geometrical conformation further impart to it "out-of-plane" degrees of freedom. In this paper we discuss the issue of intermonolayer coupling in terms of a phenomenological model that describes the necessary types of degrees of freedom and their interplay, which reflects different modes of intermonolayer coupling. Furthermore, we investigate, based on the phenomenological model, the manifestations of the intermonolayer coupling both in the lateral ordering processes of the "in-plane" degrees of freedom and in the conformational behavior of the bilayer membrane. [S1063-651X(98)05508-1]
\end{abstract}

PACS number(s): 87.22.Bt, 68.10.-m, 82.65.Dp, 87.22.As

\section{INTRODUCTION}

One structural element universal in all biological membranes is a bilayer entity, consisting of two individual monolayers that are composed of an astonishingly large number of types of amphiphilic lipid molecules. Artificial lipid bilayers may form spontaneously when one or several types of amphiphilic lipid molecules, native to biomembranes or artificially synthesized, are dispersed in an aqueous environment under a wide range of physicochemical conditions. They are the simplest model systems of biomembranes, which can both mimic, at different levels, the molecular complexity and retain some of the essential physical properties of biological lipid bilayers [1]. Our study deals with such model lipidbilayer membranes.

One of the most recognized properties of model lipid bilayers is the following: Under typical laboratory conditions, model lipid bilayers often appear fluid, as their biological counterparts do, lacking any lateral positional ordering of lipid molecules; also, they are flexible, easily (at the impact of typical thermal fluctuations) changing their surface configurations. A phenomenological model, containing an important notion of bending rigidity, was proposed by Canham [2] and Helfrich [3] to describe this property or the physics governing "external" degrees of freedom. The model treats a fluid lipid bilayer as a single incompressible surface with the elastic free energy

$$
\mathcal{H}_{\mathrm{el}}=\sigma_{0} A+\int d A\left[\frac{\kappa}{2}\left(H-H_{0}\right)^{2}+\bar{\kappa}_{g} K\right] .
$$

$A$ represents the total area of the bilayer surface and is proportional to the total number of lipid molecules composing the bilayer. Thus, if the bilayer is in equilibrium with an external lipid reservoir, $\sigma_{0}$, being conjugate to $A$, is propor-

\footnotetext{
*Present address: Laboratory of Physical and Structural Biology, National Institutes of Child Health and Human Development, National Institutes of Health, Bethesda, MD 20892.
}

tional to the chemical potential of the reservoir. The free energy also involves local surface invariants up to the second derivatives of the surface: the mean curvature $H=1 / R_{1}$ $+1 / R_{2}$ and the Gaussian curvature $K=1 / R_{1} R_{2}$, where $R_{1}$ and $R_{2}$ are the two principal radii of curvature of the surface. One of the physical parameters in the model $\kappa$ measures the degree of the membrane flexibility and is called bending rigidity. $\bar{\kappa}_{g}$ is another bending rigidity, which becomes irrelevant when the surface topology of the membrane is fixed as is often the case. The bilayer aspect is accounted for only by the constant $H_{0}$, termed "bilayer spontaneous curvature," which allows for asymmetry (difference) in either the chemical or the physical nature of the two constituting monolayers. For a single-component lipid bilayer immersed in a homogeneous environment, $H_{0}$ is assumed to be zero. Many studies based on this model have provided a great deal of insight into the conformational aspect of the thermodynamic behavior of fluid lipid bilayers $[1,4]$.

Apparently, this model neglects the effects of the different types of "in-plane" degrees of freedom that pertain to a lipid bilayer: translational degrees of freedom to describe the positions of lipid molecules within each monolayer, conformational degrees of freedom to describe the large number of conformations each lipid chain can assume, and finally, compositional degrees of freedom to describe the molecular compositions of each monolayer. On the other hand, it may be expected that under changes of thermodynamic (and chemical) conditions these different types of degrees of freedom will undergo changes in their collective behavior or lateral ordering processes and that large fluctuations associated with these degrees of freedom may arise naturally in the ordering processes, leading to in-plane heterogeneity. Indeed, biophysical studies of model lipid bilayers have been providing mounting evidence for this [1,5]. For example, a study of lipid bilayers of a binary mixture of dimyristoylphosphatidylcholine (DMPC) and distearoylphosphatidylcholine ( $\mathrm{DS}_{d 54} \mathrm{PC}$ ) based on a small-angle neutron-scattering technique [6] clearly revealed the presence of coherent domains rich in DSPC, a signature of the ordering in 
the compositional degrees of freedom. Furthermore, the critical nature of the ordering in systems of equimolar mixture of the two lipids was indicated by the divergence of a coherence length as a particular temperature was approached. Similar critical demixing processes also take place in PC-cholesterol mixtures, which are of particular interest to membrane physics [5,7]. Another important type of lateral ordering processes, known as the "main transition" or the "chainmelting transition," involve the conformational degrees of freedom and can occur even in single-component lipid bilayers. During these transitions, hydrocarbon chains of lipid molecules collectively undergo a change between a (hightemperature) disordered state, characterized by a large number of conformations, and a (low-temperature) ordered state, characterized largely by the trans conformation. It has also been demonstrated that such chain-melting processes in most model systems of lipid (with the PC headgroup and saturated acyl chains) bilayers studied appear pseudocritical, involving large fluctuations in the molecular densities in the two monolayers. Clearly, these cases necessitate the need to deal with the in-plane degrees of freedom.

Any modeling of a fluid lipid bilayer has to reflect its unique molecular architecture and properties. A lipid bilayer actually consists of two two-dimensional systems (monolayers), each of which possesses a distinct set of "internal" degrees of freedom. While the internal degrees of freedom in one monolayer can in principle behave differently from those in the other, there exists coupling between them: The fact that the two monolayers have to follow the same geometry (for surface deformations on length scales larger than the bilayer thickness) imposes one form of coupling; direct molecular interactions, which may depend on the states of the two individual sets of internal degrees of freedom, constitute another. Finally, the geometry of the bilayer surface, or the "external" degrees of freedom, is thermodynamically relevant due to the flexibility of the bilayer. Thus the thermodynamic behavior of systems of fluid lipid bilayers will be the result of a complex interplay between lateral ordering processes of relevant internal degrees of freedom and the conformations of membrane surfaces. It is the theme of our paper both to discuss, in terms of a phenomenological model, the issue of intermonolayer coupling by examining the possible modes of the coupling and to investigate, on the basis of the model, some specific manifestations of the coupling in both lateral ordering phenomena and in conformational behavior of bilayers.

Recently, a number of theoretical studies [8-17] have modeled the intermonolayer coupling with a simple form of bilinear coupling between bilayer local geometry (specifically, local curvatures) and a local difference in the two individual in-plane density or concentration fields characterizing, respectively, the physical or chemical state of the two monolayers and have further explored some of the thermodynamic consequences of this particular form of coupling. In some studies $[10-12,14]$ care has been taken to explicitly deal with the possibility that both of the monolayers may undergo ordering processes. However, any form of intermonolayer coupling arising from direct molecular interactions has been neglected, presumably based on the assertion that direct interactions may have only a weak dependence on specific states of the in-plane fields and therefore are irrel- evant to a first approximation [12]. In this paper we will explicitly take into account contributions of direct interactions to intermonolayer coupling and will argue, based on our calculations, that such contributions, even when they are weak, are relevant in determining the thermodynamic behavior of lipid bilayers.

One of the major predictions given by those studies mentioned above is that in lipid bilayers under one form or another of mechanical constraints, lateral ordering processes may often be characterized by the appearance of modulated structures. In these modulated structures, domains with both distinctly different degrees of ordering in the in-plane fields and distinctly different curvatures appear in well-defined sizes and in a spatially alternating fashion. The well-defined sizes and surface corrugations of ordered domains find no analog in lateral ordering processes in ordinary (simple) fluids and have been used to interpret ripple phases and other ordered-domain phenomena $[9,14]$. In our work we have made efforts to further the characterization of the modulated structures. In this paper we will demonstrate that, under different thermodynamic conditions, modulated structures of different characteristics may exist and that, depending on thermodynamic control parameters, the change from one type of modulated structure to another in principle may or may not correspond to thermodynamic transitions.

The studies and the results described so far are all based on mean-field analyses of the models, in which effects of thermal fluctuations both in the in-plane degrees of freedom and in the geometrical conformation of a membrane are neglected. The mean-field approximation concerning membrane conformation is certainly valid in situations where mechanical constraints are imposed on a bilayer membrane to suppress its strong conformational fluctuations. However, we have also been particularly interested in understanding how the intermonolayer coupling may manifest itself in the conformational behavior of fluid lipid-bilayer membranes when strong fluctuations in both the internal and the external degrees of freedom are present, a question that has not been seriously addressed before. To this end we have considered situations where the effect of mechanical constraints becomes vanishingly small and have necessarily extended our study beyond the scope of the mean-field approximation. In this paper we will also describe a simplified analysis based on a field-theory approach, which we have carried out to deal with fluctuation effects, and present the result of the analysis. Previously, it has already been demonstrated that a fluid lipid bilayer under no mechanical constraints will display at (often extremely) large length scales a conformational instability towards branched-polymer configurations [18], even if the effect of in-plane fluctuations is neglected. One may thus intuitively expect that strong fluctuations in in-plane degrees of freedom will only promote this conformational instability. Indeed, our analysis shows that strong in-plane fluctuations reinforce shape fluctuations and that consequently, the conformational instability sets in on much, much shorter (therefore, perhaps experimentally accessible) length scales.

\section{PHENOMENOLOGICAL MODEL}

In this section we describe a phenomenological model for fluid lipid bilayers and discuss its physical significance. The 
model emphasizes the fact that a fluid lipid bilayer is an entity of two coupled monolayers that always assume (approximately) the same geometrical conformations, but that can display different behavior as far as the in-plane degrees of freedom are concerned. As is implicitly assumed in a phenomenological description of a physical system, specific microscopic details can largely be neglected and the large number of any relevant degrees of freedom and the physics governing them may be "coarse grained" into a few local (spatially varying) fields and a small number of phenomenological parameters, respectively. Particularly in our model of a fluid lipid bilayer, Eq. (1) is taken as the basic description of the external degrees of freedom in terms of local surface curvatures, the bending rigidity, and the bilayer spontaneous curvature. Similarly, in-plane degrees of freedom within each of the two monolayers (labeled, arbitrarily, as 1 and 2) are represented by a local scalar field on the surface $\phi_{i}(\vec{x})$ and the essence of the physical mechanisms underlying their ordering processes is captured in a few physical parameters. Explicitly, the total free energy of a bilayer is given as

$$
\begin{aligned}
F_{\text {bilayer }}= & \int d A\left\{\sigma_{0}+\frac{\kappa}{2} H^{2}-\frac{\kappa \bar{C}_{0}}{2}\left(\phi_{1}-\phi_{2}\right) H+\frac{\gamma_{12}}{4} \phi_{1} \phi_{2}\right. \\
& +\frac{1}{2}\left[\frac{c}{2}\left(\vec{\nabla} \phi_{1}\right)^{2}+\frac{t}{2} \phi_{1}^{2}+\frac{g}{4 !} \phi_{1}^{4}-\mu \phi_{1}+\frac{c}{2}\left(\vec{\nabla} \phi_{2}\right)^{2}\right. \\
& \left.\left.+\frac{t}{2} \phi_{2}^{2}+\frac{g}{4 !} \phi_{2}^{4}-\mu \phi_{2}\right]\right\} .
\end{aligned}
$$

In defining the local principal curvatures, we adopt the following sign convention: Once the two monolayers are labeled as 1 and 2, the surface normal is then chosen to point from monolayer 2 to $1 ; R_{i}(i=1,2$ here denotes the two local principal directions, respectively) is defined to be positive (negative) if the corresponding local principal curve is concave (convex) with respect to the chosen surface normal.

As is apparent in Eq. (2), we have employed a canonical model, the Landau-Ginzburg $\phi^{4}$ theory [19],

$$
F_{i}=\frac{1}{2} \int d A\left\{\frac{c}{2}\left(\vec{\nabla} \phi_{i}\right)^{2}+\frac{t}{2} \phi_{i}^{2}+\frac{g}{4 !} \phi_{i}^{4}-\mu \phi_{i}\right\}, \quad i=1,2,
$$

as a generic description of any in-plane degrees of freedom under consideration [20]. The bilayer nature of the system is reflected by the possibility that each monolayer can undergo an ordering process governed by the Landau free energy $F_{i}$. It is worth emphasizing that the physical parameters in Eq. (3) should be defined as those that characterize the ordering process that takes place when the bilayer is mechanically constrained to be flat.

We have in mind specifically two types of ordering processes, to which this generic phenomenological description applies, although the description may also be relevant to other classes of phenomena such as adsorptions to membranes of small molecules (sterols, anesthetics, etc.) [21]. One type is a phase separation process in a binary mixture of lipids, in which case the following correspondance can be made:

$$
\phi_{i}=\frac{\rho_{i}-\rho_{c}}{\rho_{c}}, \quad t \propto \frac{T-T_{c}}{T_{c}},
$$

where $\rho_{i}$ is the local composition field and $\rho_{c}$ and $T_{c}$ are the composition and temperature, respectively, characterizing the critical demixing that takes place in a flat monolayer. $\mu$ is in effect the chemical potential regulating the composition and $\mu=0$ corresponds to the situation where the composition is set at the critical value. The other type of ordering processes is the chain-melting processes in single-component lipid bilayers, as described in the Introduction. Since the chain conformational change involved in the chain melting is also manifested in a corresponding change in the molecular density $\rho_{i}$, we choose the density field as the representative. The chain melting can then be approximately described by Eq. (3) also, with

$$
\phi_{i}=\left(\rho_{i}-\rho_{0}\right) / \rho_{0}, \quad \mu \propto\left(T-T_{m}\right),
$$

and $t<0$. Here $\rho_{0}$ is a properly chosen average of the two densities corresponding to the chain-ordered and the chaindisordered states and $T_{m}$ is the chain-melting temperature $[5,22]$.

The main theme of our paper, intermonolayer coupling, is specifically represented by the last two terms in the first line of Eq. (2). The first form $-\kappa \bar{C}_{0}\left(\phi_{1}-\phi_{2}\right) H / 2$ is an explicit model expression of the notion of bilayer spontaneous curvature and is based on the following reasoning. In general, any two opposing local elements of the two monolayers can be different in their chemical or physical states, represented by $\phi_{1}(\vec{x})$ and $\phi_{2}(\vec{x})$. It is thought that the different local fields imply the preferences of the two monolayers for different local mean curvatures $[12,14,23]$. Hence the constraint that the two monolayers must conform to the same local mean curvatures simply means that the different preferences of the two monolayers cannot be satisfied simultaneously, leaving either one monolayer or both frustrated. However, the degree of the frustration due to such local transverse asymmetry can be minimized if a particular local mean curvature is assumed. This particular value is the local bilayer spontaneous curvature and is approximated by $\bar{C}_{0}\left(\phi_{1}\right.$ $\left.-\phi_{2}\right) / 2$, where $\bar{C}_{0}$, having the physical dimension of the inverse length, is a phenomenological constant that depends on the material properties of the bilayer. This form of coupling is precisely the bilinear coupling that has been proposed in models similar to Eq. (2) to model the interplay between in-plane degrees of freedom and membrane conformations [8-17].

The second form of intermonolayer coupling $\gamma_{12} \phi_{1} \phi_{2}$ is introduced to describe the possibility that direct intermonolayer interactions may also depend on the physical or chemical states of the monolayers and give contributions to the total free energy of a bilayer. Molecular interactions of different origins may be responsible for this effect. For example, lipid molecules residing separately in the two monolayers interact via van der Waals interactions that depend both on the chain-conformational states and on the spatial packing of the interacting molecules and in turn on the local lateral densities of the two monolayers $\phi_{1}$ and $\phi_{2}$. The contribution from the cohesive part of van der Waals interac- 
tions may be modeled, to a first approximation, by the proposed $\gamma_{12} \phi_{1} \phi_{2}$, where $\gamma_{12}$ takes on negative values. In a bilayer containing charged lipids, electrostatic interaction may lead to coupling between the two constituent monolayers, in situations where ionic concentrations of the aqueous solutions surrounding the bilayer are very low so that the hydrocarbon interior of the bilayer appears "transparent" to electrostatic fields [24]. In this case, $\phi_{1}$ and $\phi_{2}$ may represent, respectively, the densities or concentrations of the charged lipids in the two monolayers and $\gamma_{12}$ can be either positive or negative if the charged lipids in the two monolayers carry electric charges of either the same or opposite signs, respectively. There is experimental evidence that intermonolayer coupling of this nature is relevant in bilayers formed from lipids with charged head groups [25]. Thus, for a given system of lipid bilayer, the phenomenological parameter $\gamma_{12}$ will contain contributions from the different sources and its specific value will depend both on molecular details and packing properties of the consitituent lipids and on solution properties of the aqueous environment surrounding the bilayer. Conventional approximations have so far neglected this form of direct interactions between monolayers [12,14], based on the argument that such direct interactions may not be significant in their strength compared to other relevant effects such as bending and that neglecting them will not therefore lead to qualitative changes in our understanding of the systems [12]. However, we will demonstrate with our study that this coupling, even when it is weak, plays a nontrivial role in determining the characteristics of in-plane ordering processes in a bilayer.

The last physical parameter in Eq. (2) to comment on is $\sigma_{0}$. It has the physical dimension of energy per unit area, that of surface tension, i.e., it measures the energy cost of increasing some area of a bilayer. Its definition and physical interpretation depend on what area is being considered. If the area refers to the total area of a lipid bilayer in equilibrium with some external reservoir of the lipid molecules, $\sigma_{0}$ is simply proportional to the chemical potential of the reservoir. However, only when the bilayer is subject to mechanical constraints [26] does $\sigma_{0}$ become a relevant parameter in determining the thermodynamic behavior of the bilayer: It has been understood that no matter what $\sigma_{0}$ is, a freely suspended (i.e., under no mechanical constraints) fluid membrane always appears crumpled on large length scales [2729]. When mechanical constraints are present, $\sigma_{0}$ may be related to the strength of the mechanical force needed to enforce the constraints. There are different ways of implementing mechanical constraints on a fluctuating fluid bilayer, such as those discussed in [26] or that of confining the bilayer between two walls [12]. In this paper we choose, for the sake of simplicity, to represent mechanical constraints with a planar frame of a total area $A_{p}$, which the bilayer spans, and we carry out our calculations in this representation. It turns out that if $\sigma_{0}$ exceeds some threshold value (which depends on other physical parameters such as temperature $\kappa$, etc.), the mechanical constraint can be applied with a finite mechanical force (see below) and the bilayer in its thermodynamic equilibrium state assumes a certain extended, instead of "crumpled" mean conformation. The area of this mean conformation $A_{m}$ may be different from $A_{p}$, but scales with it linearly, and it is also different from the mean value of the total area of the bilayer $\langle A\rangle$ as a consequence of thermal fluctuations of the bilayer surface about the mean conformation. The mechanical force $\tau$ can be related to the amount of mechanical work $\tau \Delta A_{p}$ required to change the frame area by $\Delta A_{p}$ (in turn, change $A_{m}$ ) and is often called frame tension [26,30]. Its magnitude must be larger than $\sigma_{0}$ in the presence of surface fluctuations and also has a specific dependence on $\sigma_{0}$ [31]. Equivalently, an effective tension may be associated with the area of the mean bilayer conformation $A_{m}$, which is also related to the "bare" $\sigma_{0}$. Since most of our work only concerns the determination of mean bilayer conformations and does not explicitly treat the thermal fluctuations of the bilayer surface, the "surface tension" that we will refer to should be thought of as the effective tension, denoted by $\bar{\sigma}_{0}$, that is conjugate to the area of the mean equilibrium conformation. When in-plane fields and their ordering become relevant, such an effective tension contains also contributions from the ordering of the in-plane fields. To make the contributions of the in-plane fields explicit, we define $\bar{\sigma}_{0}$ to be the effective tension in a bilayer state where the in-plane fields are disordered. It is important to keep in mind that this effective tension contains the entropic contributions from all the degrees of freedom associated with the membrane surface and therefore depends on temperature and the bare physical parameters controlling those degrees of freedom.

Our last remark on the phenomenological model (2) concerns the different types of symmetry it possesses. First of all, the physics of a bilayer must be invariant under translations and rotations in the three-dimensional Euclidean embedding space. This symmetry is reflected in the fact that the surface-related quantities present in the model are invariants such as the total surface area and the mean surface curvature. Second, Eq. (2) is invariant under the "relabeling" or "inversion" of the two monolayers, which is formulated as $\left\{\phi_{1} \leftrightarrow \phi_{2}, H \rightarrow-H\right\}$ and will be referred to as $\mathcal{O}_{1}$ henceforth. This symmetry is simply a formal expression of the statement that the two monolayers composing the bilayer are considered to be subject to identical macroscopic physical and chemical conditions and that their phenomenological physical properties are considered identical as well. Situations where this symmetry is absent have been considered in other studies [9,32], but are outside the scope of the present paper. One of the most interesting thermodynamic consequences of the model (2) concerns this symmetry: Under certain conditions the two monolayers actually acquire different macroscopic states, despite the symmetry of the free energy. In other words, the symmetry may be spontaneously broken.

In addition to the above two types of principal symmetry, the phenomenological model (2) also possesses various additional types of invariances, which hold as different physical parameters are set to zero. When $\mu$ is zero, the "reduced" form of Eq. (2) is invariant under an operation $\mathcal{O}_{2}$, defined as $\left\{\phi_{1} \rightarrow-\phi_{1}, \phi_{2} \rightarrow-\phi_{2}, H \rightarrow-H\right\}$. The origin of this invariance lies partly in the formal symmetry of the Landau-Ginzburg free energy [Eq. (3)] with respect to transformation $\left\{\phi_{i} \rightarrow-\phi_{i}\right\}$ when $\mu=0$. This formal symmetry is, within the framework of the $\phi^{4}$ theory, a statement about the fact that there are two coexisting phases below a critical 
point. $\mathcal{O}_{2}$, together with $\mathcal{O}_{1}$, forms another operation $\mathcal{O}_{3}$ $=\mathcal{O}_{1} \mathcal{O}_{2}=\mathcal{O}_{2} \mathcal{O}_{1}=\left\{\phi_{1} \rightarrow-\phi_{2}, \phi_{2} \rightarrow-\phi_{1}, H \rightarrow H\right\}$, which also leaves Eq. (2) invariant when $\mu$ is set to zero [33].

Finally, another observation concerning symmetry may help us understand the role of the parameter $\gamma_{12}$, which characterizes the strength of direct intermonolayer interactions. It is easy to see that for a bilayer constrained to be in the flat configuration (conforming to the frame), the two constituting monolayers become independent of each other when $\gamma_{12}=0$. Consequently, when $\mu=0$, the effective $(H=0)$ free energy respects the symmetry of each of the monolayer free energies with respect to $\left\{\phi_{i} \rightarrow-\phi_{i}\right\}$, i.e., is invariant under the following two transformations: $\left\{\phi_{1} \rightarrow-\phi_{1}, \phi_{2}\right.$ $\left.\rightarrow \phi_{2},\right\}$ and $\left\{\phi_{1} \rightarrow \phi_{1}, \phi_{2} \rightarrow-\phi_{2},\right\}$. A nonzero $\gamma_{12}$ explicitly breaks this symmetry. We will elaborate on the point in more detail when we present and discuss our results.

We end this section of description of our phenomenological model with an alternative expression of Eq. (2), which will be more convenient to use in our calculations and is written in terms of $\phi \equiv\left(\phi_{1}-\phi_{2}\right) / 2$ and $\psi \equiv\left(\phi_{1}+\phi_{2}\right) / 2$ :

$$
\begin{aligned}
F_{\text {bilayer }}= & \int d A\left\{\sigma_{0}+\frac{\kappa}{2}\left(H^{2}-2 \bar{C}_{0} \phi H\right)+\frac{c}{2}(\vec{\nabla} \phi)^{2}+\frac{t_{\Delta}}{2} \phi^{2}\right. \\
& +\frac{g}{4 !} \phi^{4}+\frac{c}{2}(\vec{\nabla} \psi)^{2}+\frac{t_{\Delta}+\gamma_{12}}{2} \psi^{2} \\
& \left.+\frac{g}{4 !} \psi^{4}+\frac{g}{4} \phi^{2} \psi^{2}-\mu \psi\right\}
\end{aligned}
$$

where $t_{\Delta} \equiv t-\gamma_{12} / 2$. We will sometimes refer to $\phi$ and $\psi$ as the "difference field" and the "average field," respectively.

\section{MEAN-FIELD CALCULATIONS: LATERAL ORDERING IN FLUID LIPID BILAYERS}

In this section we focus on one aspect of the thermodynamic behavior of a fluid lipid bilayer (lateral ordering processes) to elucidate some of the thermodynamic consequences of the intermonolayer coupling mechanisms discussed in the preceding section. Our calculations are based on "mean-field" considerations, which assume that the thermodynamic state of a bilayer is determined by minimizing the free energy given in Eq. (6) with respect to both the external and the in-plane fields. In other words, we not only neglect the effects of strong in-plane fluctuations, but also do not explicitly treat the conformational fluctuations of the bilayer. Furthermore, we mostly consider situations in which the mean equilibrium geometrical conformation of the bilayer is, if it is indeed not flat, not too far from the flat configuration. These considerations about the external degrees of freedom are valid when $\bar{\sigma}_{0}$ is sufficiently large.

There are a number of physical parameters in the model [Eq. (6)] to be dealt with: $\bar{\sigma}_{0}, t_{\Delta}, \gamma_{12}, g$, and $\mu$, which all have the physical dimension of surface energy density; $\kappa$ and $c$, which have the unit of energy; and $\bar{C}_{0}$, the inverse of which sets a length scale in the problem. It turns out that the precise values of $c$ and $g$ do not influence the qualitative features of the thermodynamic behavior of the model. Hence, for computational convenience, they are set to 1. Fol- lowing this convention, $\sqrt{c / g}$, having the physical dimension of length, then becomes the normalization unit for all length scales in the problem. This implies that henceforth, all physical parameters should be considered dimensionless, normalized by the chosen energy unit $c$ and the length unit $\sqrt{c / g} . \kappa$ and $\bar{C}_{0}$ are considered to take on some fixed values, from which we obtain a (normalized) length scale $\lambda_{\mathrm{sc}}$, where $\lambda_{\mathrm{sc}}^{-2} \equiv \kappa \bar{C}_{0}^{2}$. The inverse of $\lambda_{\mathrm{sc}}$ may then be thought of as an effective bilayer spontaneous curvature. We will thus consider situations where $t_{\Delta}, \gamma_{12}, \mu$, and $\bar{\sigma}_{0}$ are the relevant control parameters.

Specifically, we have in mind a bilayer membrane that spans a flat frame of area $A_{p}$ and assumes a nearly flat equilibrium configuration. Hence the external degrees of freedom can be formulated precisely in terms of

$$
\vec{R}=(x, y, Z(x, y))=(\vec{x}, Z(\vec{x})),
$$

where $\vec{x}=(x, y)$ are coordinates designated to the flat frame and $Z(x, y)$ is the deviation from the flat configuration. Both the surface area and the mean curvature of the bilayer can then be expressed in terms of the first and second derivatives of $Z$ and expanded in a power series ordered by $Z$. $Z(x, y)$ is considered small enough (for nearly flat configurations) so that in the following calculations a harmonic approximation will be used in treating $Z(x, y)$, in which the surface-related terms in the free energy are approximated only by terms that are quadratic in $Z$.

The minimization of the free energy (6) can be performed conveniently in the Fourier space where all the fields are expressed in terms of their Fourier components

$$
\begin{aligned}
& Z(\vec{x})=\int \frac{d^{2} q}{(2 \pi)^{2}} Z(\vec{q}) e^{i \vec{q} \cdot \vec{x}}, \\
& \phi(\vec{x})=\int \frac{d^{2} q}{(2 \pi)^{2}} \phi(\vec{q}) e^{i \vec{q} \cdot \vec{x}}, \\
& \psi(\vec{x})=\int \frac{d^{2} q}{(2 \pi)^{2}} \psi(\vec{q}) e^{i \vec{q} \cdot \vec{x}} .
\end{aligned}
$$

Minimization with respect to $Z(\vec{q})$ leads straightforwardly to the relationship between the equilibrium bilayer deformation and the equilibrium configuration of the in-plane difference field $\phi$,

$$
Z(\vec{q})=\frac{-\kappa \bar{C}_{0}}{\kappa q^{2}+\bar{\sigma}_{0}} \phi(\vec{q})
$$

Substituting this relationship into the free energy (6) yields

$$
F_{\text {bilayer }}=\bar{\sigma}_{0} A_{p}+f_{\text {eff }} A_{p}
$$

where $f_{\text {eff }}$ is an effective free energy density associated with the in-plane fields $\phi(\vec{q})$ and $\psi(\vec{q})$, given by 


$$
\begin{aligned}
f_{\mathrm{eff}} A_{p}= & \frac{1}{2} \int \frac{d^{2} q}{(2 \pi)^{2}}\left[p_{\phi}(\vec{q}) \phi(\vec{q}) \phi(-\vec{q})\right. \\
& \left.+p_{\psi}(\vec{q}) \psi(\vec{q}) \psi(-\vec{q})\right] \\
& +\int d x d y\left[\frac{1}{4 !} \phi^{4}+\frac{1}{4 !} \psi^{4}+\frac{1}{4} \phi^{2} \psi^{2}-\mu \psi\right] .
\end{aligned}
$$

$p_{\phi}(\vec{q})$ and $p_{\psi}(\vec{q})$ have the expressions, respectively,

$$
p_{\phi}(\vec{q})=t_{\Delta}+q^{2}-\frac{\lambda_{\mathrm{sc}}^{-2} q^{2}}{q^{2}+\xi_{c}^{-2}}, \quad p_{\psi}(\vec{q})=t_{\Delta}+\gamma_{12}+q^{2},
$$

where $\xi_{c}=\sqrt{\kappa / \bar{\sigma}_{0}}$ is a length scale determined by two competing physical effects, the bending rigidity and the surface tension.

Equation (11) shows that in the presence of the bilinear coupling term, removing the "out-of-plane" degrees of freedom introduces an extra contribution to $p_{\phi}(\vec{q})$, as expressed by the last term in $p_{\phi}(\vec{q})$. One of the most important consequences of this effect is that, when the lateral ordering process, for example, the phase separation, takes place, the ordered phases are often not macroscopically homogeneous, but rather appear to be spatially modulated, i.e., consisting of domains that alternate between the two coexisting ordered states and have characteristic sizes. The mechanism underlying this appearance of specific length scales in lateral ordering processes can be revealed by analyzing both Eqs. (11) and (12) (for the case of $\mu=0$, for simplicity). Mean-field solutions for the two fields $\phi$ and $\psi$ are given by minimizing $f_{\text {eff }}$ with respect to both of the fields and the signs of $p_{\phi}(\vec{q})$ and $p_{\psi}(\vec{q})$ primarily determine whether nonzero solutions for $\phi$ or $\psi$ exist. Since $p_{\phi}(\vec{q})$ and $p_{\psi}(\vec{q})$ are proportional to the reduced temperature $t \sim T-T_{c}$ when other parameters $\lambda_{\mathrm{sc}}, \xi_{c}$, and $\gamma_{12}$ are held fixed, reducing the temperature amounts to decreasing $p_{\phi}(\vec{q})$ and $p_{\psi}(\vec{q})$. At high temperatures, both $p_{\phi}(\vec{q})$ and $p_{\psi}(\vec{q})$ are positive; consequently, both $\phi$ and $\psi$ are zero, corresponding to a flat, completely disordered $(D)$ state. As the temperature is lowered, the minimum of either $p_{\phi}(\vec{q})$ or $p_{\psi}(\vec{q})$ reaches zero first at a particular temperature and then becomes negative, leading to nonzero solutions of the corresponding field. This particular temperature marks the onset of instability of the flat and disordered state of the bilayer with respect to the ordering of either the $\phi$ field or the $\psi$ field. The presence of the extra term in $p_{\phi}(\vec{q})$ imparts a nontrivial characteristics to the ordering of $\phi$ field: In a weak-tension regime, where $\xi_{c}^{2} / \lambda_{\mathrm{sc}}^{2}>1$ or $0<\bar{\sigma}_{0}<\bar{\sigma}_{0}^{*}$ where

$$
\bar{\sigma}_{0}^{*}=\kappa^{2} \bar{C}_{0}^{2},
$$

the minimum of $p_{\phi}(\vec{q})$ occurs at a nonzero $q_{0}$ given by

$$
q_{0}^{2}=\xi_{c}^{-2}\left(\sqrt{\xi_{c} / \lambda_{\mathrm{sc}}}-1\right) .
$$

Hence a specific length scale $1 / q_{0}$ is associated with the appearance of nonzero solutions of $\phi$. In other words, the ordering of $\phi$ proceeds through the appearance of ordered domains of particular size $1 / q_{0}$.

The basic physical forces driving this appearance of ordered domains of well-controlled sizes are also apparent to see. The length scale $\xi_{c}$ defined above is in fact a length scale for crossover: The bending rigidity $\kappa$, when $\phi$ becomes ordered (nonzero), tends to bend the bilayer towards the nonzero spontaneous curvature $\left(\bar{C}_{0} \phi\right)$ in order to minimize the bending energy and is more effective on short length scales, while $\bar{\sigma}_{0}$ more effectively controls deformations of long wavelengths and tends to keep the bilayer flat on large length scales. $\xi_{c}$ separates these two different regimes of length scales. The compromise between these two competing effects leads to the selection of the specific length scale $1 / q_{0}$. Similar mechanisms have been discussed in other studies $[9,12,14]$.

In the actual determination of the phase diagrams, we focus on four types of principal states that are obvious to consider: (i) the flat, disordered (D) state, characterized by $\phi=0$ and $\psi=0$; (ii) a flat, homogeneously ordered (HO1) state, represented by

$$
\phi=0, \quad \psi=\text { const } \neq 0,
$$

where there is no bilayer transverse asymmetry, i.e., where the two monolayers are in exactly the same ordered state; (iii) a flat, homogeneously ordered ( $\mathrm{HO} 2)$ state, represented by

$$
\phi=\text { const } \neq 0, \quad \psi=\text { const }
$$

in which the two monolayers actually assume different states as a nonzero value of the $\phi$ field indicates; in other words, the bilayer develops a global transverse asymmetry; and (iv) curved, spatially modulated (M) states, described by

$$
\phi=\phi^{(1)} \cos \left(\bar{q}_{0} x\right), \quad \psi=\psi^{(0)}+\psi^{(2)} \cos \left(2 \bar{q}_{0} x\right),
$$

where the bilayer acquires a local transverse asymmetry, in contrast with the $\mathrm{HO} 2$ state.

The description of the field configurations in the modulated states given in Eq. (17) is an ansatz. The basic reason for using this ansatz lies in the length-scale selection associated with the ordering of the $\phi$ field. The part of the ansatz for the $\psi$ field follows from an observation of the nonlinearity of the effective free energy (11) or, more specifically, of the term $\phi^{2} \psi^{2}$ in Eq. (11). If the sum field $\psi$ were expressed in terms of the cosine series of period $\bar{q}_{0}, \phi^{2} \psi^{2}$ would yield a nontrivial term involving the lowest modes $\left(\phi^{(1)}\right)^{2} \psi^{(0)} \psi^{(2)}$. It is not difficult to see that when $\phi^{(1)}$ becomes nonzero, having nonzero $\psi^{(0)}$ and $\psi^{(2)}$ of opposite signs may lead to lower free energy. Thus we propose the ansatz to include this possibility. The numerical evidence presented in Ref. [14] also supports this ansatz. Another related point is that in our ansatz the wave number of the modulation $\bar{q}_{0}$ is also considered as a variational variable, along with all the relevant amplitudes. Finally, our ansatz implies that the domains in the modulated states appear in the form of stripes. In other words, we do not expect hex- 


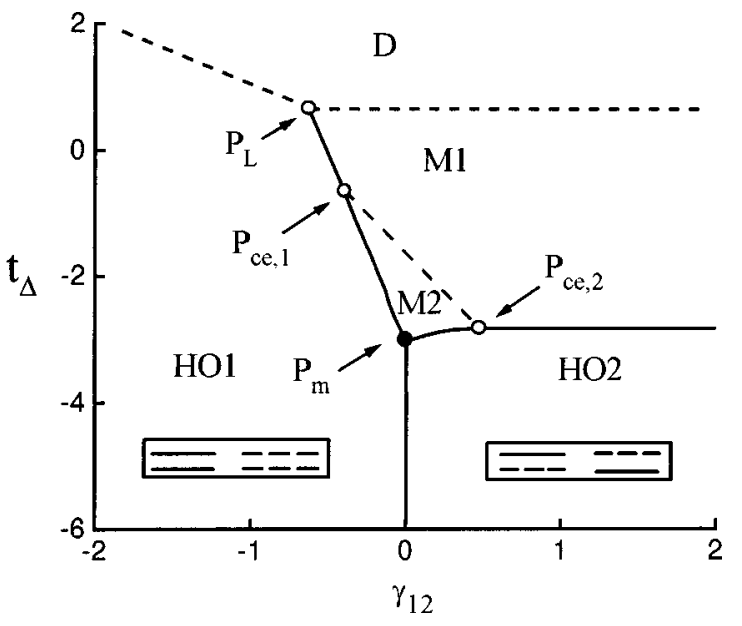

FIG. 1. Mean-field phase diagram in the parameter space spanned by $t_{\Delta}$ and $\gamma_{12}$, for $\xi_{c}^{-2} \equiv \sigma_{0} / \kappa=0.04, \lambda_{\text {sc }}^{-2}=1$, and $\mu=0$. The labeling of the phases is the same as defined in the text. Note that the M1 region is actually a region of two-phase coexistence, the M2 region that of four-phase coexistence, the HO1 region that of two-phase coexistence, and the $\mathrm{HO} 2$ region also that of two-phase coexistence. Dashed lines represent lines of second-order phase transitions; solid lines represent lines of first-order transitions, in particular, the line of $\gamma_{12}=0$ is a line of four-phase (illustrated in the cartons where solid lines and dashed lines represent the two degenerate states each of the two monolayer fields can assume) coexistence. Open circles denote critical points (a Lifshitz point $P_{L}$, two critical end points $P_{\text {ce, } 1}$ and $\left.P_{\text {ce, } 2}\right)$; filled circles denote points of multiple-phase coexistence $\left(P_{m}\right)$. Analytical expressions for some of the transition lines are available and are given in the Appendix, as well as the coordinates of the special points.

agonal or circular domains to appear. Such modulated phases were predicted in both Refs. [9] and [32] only for bilayer systems where thermodynamic or chemical conditions explicitly impose a bilayer transverse asymmetry; in other words, the phenomenological free energies for those systems will no longer have the symmetry under the bilayer inversion $\left(\mathcal{O}_{1}\right)$. Such cases are not considered in this paper.

The relative energetics of the types of principal states described above are then calculated, numerically and analytically whenever possible, as different control parameters are varied. The results of the calculations are summarized in the following series of two-dimensional phase diagrams where two specific physical parameters are chosen as the control parameters [34]. Analytical expressions that can be obtained for certain phase boundaries and special points are relegated to the Appendix.

Figure 1 is a phase diagram illustrated in the parameter space spanned by $t_{\Delta}$ and $\gamma_{12}$, but for a fixed value of $\lambda_{\mathrm{sc}}^{-2}$ $=1$ and a fixed value of $\xi_{c}^{-2} \equiv \bar{\sigma}_{0} / \kappa=0.04$ [36] and for the special value of $\mu=0$. This phase diagram serves to illustrate the basic predictions from our study of the phenomenological model (6). All the principal states under our considerations appear in this phase diagram as equilibrium phases. The physics underlying the appearance of these different phases in different regions of the parameter space can largely be understood intuitively. The flat, disordered (D) phase appears in the high-temperature region. The low-temperature HO1 phase, where the two monolayers are in an identical ordered state, shows up in the region where the direct inter- monolayer interaction is attractive $\left(\gamma_{12}<0\right)$ and relatively strong, for such an interaction favors commensuration of the two monolayers. As this direct interaction becomes less attractive and then repulsive, the energetic requirement for one monolayer to behave commensurately with the other becomes less stringent; consequently, either a modulated M phase, where the bilayer develops a local transverse asymmetry, or the $\mathrm{HO} 2$ phase, where the bilayer acquires a global transverse asymmetry, becomes the equilibrium phase, depending on both the interaction and the temperature.

The presence and the relative energetics of the $\mathrm{D}, \mathrm{M}$, and HO1 states have already been largely understood for the special case where $\gamma_{12}=0$, due to the previous studies reported in Refs. [12] and [14]. However, our study reveals two new results. First, there can exist more than one type of modulated bilayer structures as distinct thermodynamic phases and thermodynamic singularities may arise, associated with the transitions between the different modulated structures. In particular, by adopting the ansatz given in Eq. (17), we have discovered that, for a range of values of $0<\xi_{c}^{-2}<\xi_{c, M}^{-2}$ (see the Appendix for the definition of $\xi_{c, M}^{-2}$ ), a line of secondorder transitions (ending at two critical end points, $P_{\mathrm{ce}, 1}$ and $\left.P_{\mathrm{ce}, 2}\right)$ exists, separating two distinct types of modulated structures: a M1 structure, where $\phi^{(1)}$ is nonzero, but $\psi=0$, i.e., where the modulation is solely associated with the difference field, and a M2 structure, where the average field $\psi$ also becomes nonzero and modulated (at half of the wavelength for $\phi$ ), with nonvanishing amplitudes of $\psi^{(0)}$ and $\psi^{(2)}$ [37]. Schematic representations of the surface conformation and the in-plane states of a bilayer in these two types of modulated phases are given in Fig. 2. For $\xi_{c}^{-2}>\xi_{c, M}^{-2}$, the region of the M2 structure disappears and the two critical end points merge with the multiple-phase coexistence point $P_{m}$.

Second, $\gamma_{12}$, representing some direct intermonolayer interactions, plays a relevant role, even when it is small, in determining the thermodynamic behavior of a bilayer. As Fig. 1 shows, in the low-temperature region, $\gamma_{12}=0$ is actually a line of coexistence of four degenerate phases, the two $\mathrm{HO} 1$ phases and the two $\mathrm{HO} 2$ phases, as a result of the last type of additional symmetry discussed in Sec. II. A nonzero $\gamma_{12}$, however small, performs the role of a "symmetrybreaking field" and removes this degeneracy: When $\gamma_{12}<0$, the HO1 phases are the equilibrium phases; when $\gamma_{12}>0$, the $\mathrm{HO} 2$ phases become the equilibrium phases, where the bilayer transverse symmetry is spontaneously broken.

Similarly, both the second-order D-M1 and M1-M2 transitions can be put in the context of symmetry (see Sec. II). It is easy to see that, at the D-M1 transitions, the symmetry of the free energy (6) under the operations represented by $\mathcal{O}_{1}$ and $\mathcal{O}_{2}$ is spontaneously broken, while the symmetry under the operation of $\mathcal{O}_{3}$ is still respected by the M1 structure. The second-order transition between the M1 and the M2 structures finally breaks the $\mathcal{O}_{3}$-associated symmetry.

Situations where the "chemical potential" $\mu$ is nonzero are perhaps more often encountered than the special case of $\mu=0$. In Fig. 3 we display a collection of six phase diagrams illustrated in the parameter space spanned by $t_{\Delta}$ and $\mu$, for six different values of $\gamma_{12}$, respectively. The values of $\lambda_{\mathrm{sc}}^{-2}$ and $\xi_{c}^{2}$ are the same as those used for obtaining Fig. 1. Each 


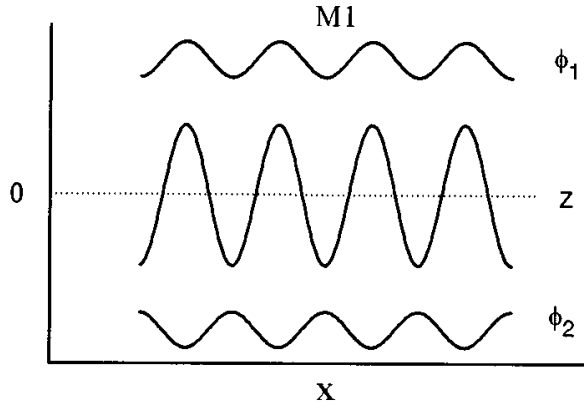

(a)

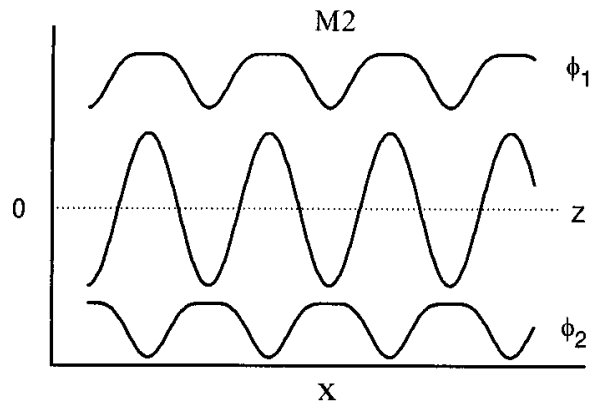

(b)

FIG. 2. Schematic illustration of the corresponding surface conformation $(Z)$ and individual profiles of the two in-plane fields $\left(\phi_{1}\right.$ and $\phi_{2}$ ) of a bilayer in (a) the M1 structure and (b) the M2 structure. The local bilayer transverse asymmetry is indicated by the phase shift in the profiles of $\phi_{1}$ and $\phi_{2}$.

of these phase diagrams has its distinct topology and a change in the value of $\gamma_{12}$ may lead to the evolution of the topology of the $t_{\Delta}-\mu$ phase diagram from one type to another. This figure again demonstrates the relevance of the direct intermonolayer interactions. Several characteristic features of the $t_{\Delta^{-}} \mu$ phase diagrams can be related to the phase diagram in Fig. 1. In most of the six phase diagrams, the second-order transition from the flat, disordered D to the modulated $\mathrm{M}$ phases persists over a range of small values of $\mu$ and can be seen as the evolution of the second-order D-M1 transition in Fig. 1 as $\mu$ becomes nonzero. The HO1-M transition becomes first order at the two tricritical points $P_{\mathrm{ce}, 1}$ and $P_{\mathrm{ce}, 2}$. This mechanism is the same as that discussed in Ref. [12]. The critical point $P_{c, M}$ terminating a line of twoM2-phase coexistence directly corresponds to the secondorder M1-M2 transition line in Fig. 1. Finally, the lines of coexistence between the $\mathrm{HO} 1$ and $\mathrm{HO} 2$ phases in the phase diagrams shown in Figs. 3(e) and 3(f) are also the direct consequence of the four-phase coexistence line in Fig. 1 [as also shown in Fig. 3(d)].

The phase diagram [Fig. 3(d)] for the special case where $\gamma_{12}=0$ deserves further comments on its relation to the phase diagrams obtained in some of the previous works $[12,14]$, which deal with only the situation of $\gamma_{12}=0$. The gross topology of the phase diagram in Fig. 3(d) is similar to those of the previously obtained phase diagrams. However, two specific features can be distinguished in Fig. 3(d): First, there exists a line of the first-order transitions between two modulated phases (of the M2 nature) that ends in a critical point $P_{c, M}$; second, below the point of multiple-phase coexistence $P_{m}$ two $\mathrm{HO} 2$ phases also exist on the $\mu=0$ line, in addition to the two $\mathrm{HO} 1$ phases that have already been predicted [12].

What is also clear from Fig. 3 is that the M2 structure is the more prevalent form of modulation predicted by the model, occupying considerable regions in the parameter space. One characteristic of this structure is that the wavelength of modulation $1 / \bar{q}_{0}$ varies with the control parameters, in contrast to the M1 structure, in which $\bar{q}_{0}$ remains fixed at the value given by Eq. (14).

Of course, the phase diagrams presented above do not imply the absolute thermodynamic stability of the considered principal phases. In other words, there may exist in some regions of the parameter space other types of phases that may have lower free energy than those we have considered. One likely candidate of such other phases would be a phase of "vesicle chains" (if the bilayer surface is thought to keep its topology of a single connected surface). It could be imagined that this phase may have lower free energy in regions of low temperatures and low effective surface tensions, than the $\mathrm{HO} 1$ and the $\mathrm{HO} 2$ phase, for example. In fact, the relative thermodynamic stability of lamellar phases (HO1-like) to vesicle phases has been explored in Ref. [11].

At the outset of this section we pointed out that our meanfield calculations are only valid when a "sufficiently large" $\bar{\sigma}_{0}$ is present. We end this section with a remark on the breakdown of the mean-field considerations. Our mean-field calculations state that the free energy density of an equilibrium state is given by [see Eq. (10)]

$$
f_{\text {bilayer }} \equiv \frac{F_{\text {bilayer }}}{A_{p}}=\bar{\sigma}_{0}+f_{\text {eff }}\left(\bar{\sigma}_{0} / \kappa, \lambda_{\mathrm{sc}}^{-2}, t_{\Delta}, \gamma_{12}, \mu\right) \text {, }
$$

where $f_{\text {eff }}$ is the equilibrium contribution from the in-plane fields and is always negative (the disordered phase corresponds to the zero value). Hence there will be loci in the parameter space where $f_{\text {bilayer }}$ becomes zero. For example, we may imagine a situation in which all parameters but $t_{\Delta}$ are fixed. Reducing $t_{\Delta}$ leads to more and more negative values of $f_{\text {eff }}$, and at some particular value of $t_{\Delta}$, the negative contribution from the in-plane fields cancels out $\bar{\sigma}_{0}$. Hence the mean-field theory itself suggests that below this point the mean-field state is no longer thermodynamically stable. It is certain, therefore, that in the absence of mechanical constraints the mean-field approach does not apply.

\section{FIELD-THEORY CALCULATION: CONFORMATIONAL INSTABILITY}

The purpose of this section is again to demonstrate the importance of the intermonolayer coupling, in particular, the aspect represented by the bilinear coupling between membrane conformation and the in-plane difference field, by examining the manifestation of the coupling in the conformational behavior of fluid lipid bilayers. The mean-field calculations presented in the preceding section require that there should be sufficiently strong mechanical force or "sufficiently large" $\bar{\sigma}_{0}$ to suppress strong conformational fluc- 


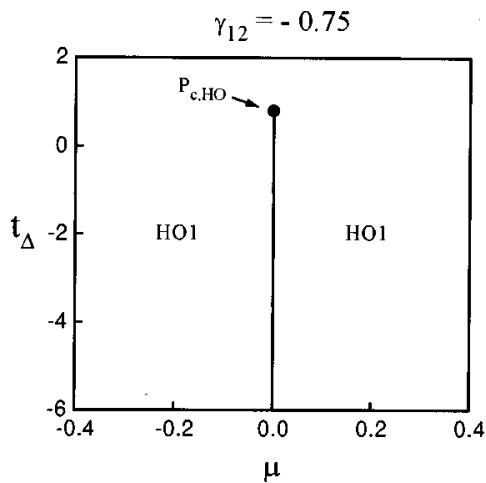

(a)

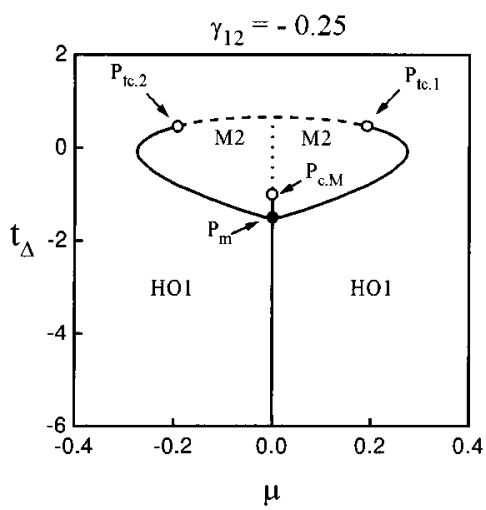

(c)

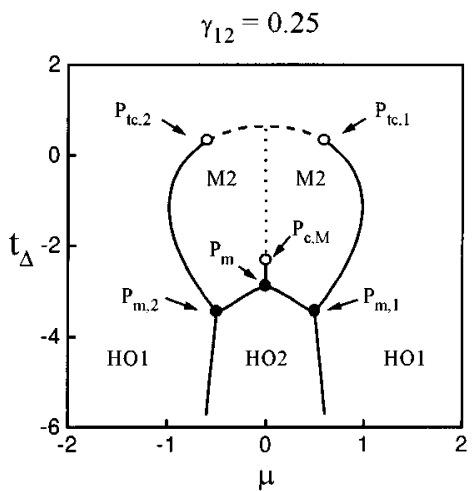

(e)

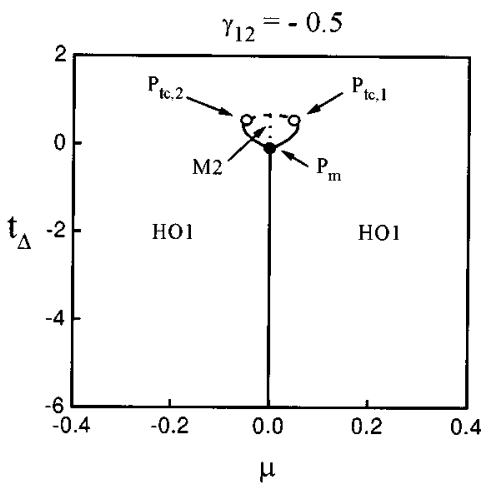

(b)

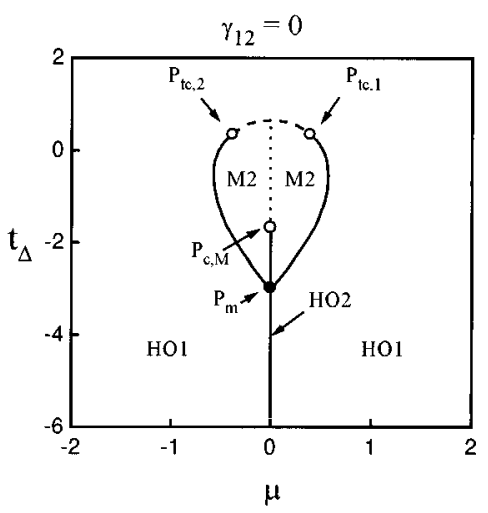

(d)

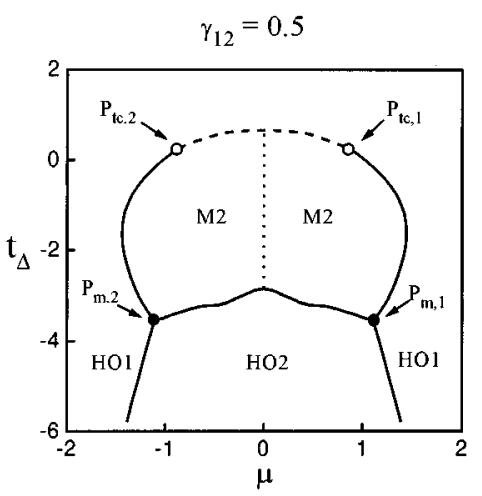

(f)
FIG. 3. Collection of six phase diagrams in the parameter space spanned by $t_{\Delta}$ and $\mu$, calculated correspondingly for six different values of $\gamma_{12}:$ (a) $\gamma_{12}=-0.75$, (b) $\gamma_{12}=-0.5$, (c) $\gamma_{12}=$ -0.25 , (d) $\gamma_{12}=0$, (e) $\gamma_{12}=0.25$, and (f) $\gamma_{12}$ $=0.5 . \xi_{c}^{-2}=0.04$ and $\lambda_{\mathrm{sc}}^{-2}=1$ are again fixed, as in Fig. 1. The conventions for labeling the phases, phase boundaries, and special points are the same as those used in Fig. 1. In addition, dotted lines are used to indicate the loci in the parameter space where the corresponding modulated structures are of the M1 type, although these loci are not phase boundaries. Three types of critical points appear in these phase diagrams: two tricritcal points $P_{\mathrm{tc}, 1}$ and $P_{\mathrm{tc}, 2}$, a critical point terminating the line of coexistence between two HO1 states $P_{c, \mathrm{HO}}$, and a critical point terminating the line of coexistence between two M2 states. Points of multiple-phase coexistence are $P_{m}, P_{m, 1}$, and $P_{m, 2}$. Analytical expressions for the two tricritical points are given in the Appendix. Note that only the $\mathrm{M} 2$ and the $\mathrm{HO} 2$ regions in these phase diagrams are regions of (twophase) coexistence, corresponding to the degeneracy of the states in $\phi$ and $-\phi$. Note also the difference between the scale of the $\mu$ axes used in (a)-(c) and that used in (d)-(f). tuations of a fluid membrane and keep it in a nearly flat equilibrium configuration. In that case, the conformational behavior of the membrane appears "trivial." In this section we extend our analysis beyond the scope of the mean-field theory and consider limit situations when $\bar{\sigma}_{0}$ approaches 0 , i.e., when mechanical constraints are no longer enforced.

Such situations are particularly interesting. As we have already (briefly) mentioned (see Sec. II), a fluid membrane under no mechanical constraints does not maintain extended conformations, or rather, appears crumpled, on large length scales even when the in-plane degrees of freedom are not relevant. In fact, there exists a specific length scale $\xi_{p}$, the persistence length, beyond which the bilayer surface loses correlation in its local orientations [38]. In other words, $\xi_{p}$ separates two distinct regimes of length scales $(l)$, corresponding to two types of membrane conformational behav- ior: (a) On small length scales $l \leqslant \xi_{p}$, the bending rigidity is in control and the membrane appears flat (if $H_{0}=0$ ) and is stable against thermal fluctuations and (b) on large length scales $l \gg \xi_{p}$, conformational entropy dominates and the membrane surface collapses into a large collection of strongly fluctuating "'branched-polymer"'-like configurations [18].

The physical mechanism underlying the crumpling of a fluid membrane is purely entropic and lies in the nonlinearity inherent in the bending elastic energy in Eq. (1), which leads to interactions between bending modes of different wavelengths. The consequence of such interactions is that the effective bending rigidity governing a long-wavelength $(l)$ bending mode is actually smaller than that controlling a shorter-wavelength $\left(l_{0}\right)$ bending mode. In other words, the membrane appears "softer" on large length scales. Statisti- 
cal mechanical analysis of Eq. (1) has provided a quantitative expression of this entropic effect [27,28]:

$$
\kappa_{e, 0}(l)=\kappa\left(l_{0}\right)-\frac{3 k_{B} T}{4 \pi} \ln \left(\frac{l}{l_{0}}\right) .
$$

An estimate of the persistence length, which has been confirmed by computer-simulation studies [39], follows immediately from Eq. (19),

$$
\xi_{p} \simeq l_{0} \exp \left(\frac{4 \pi \kappa\left(l_{0}\right)}{3 k_{B} T}\right),
$$

corresponding to the length scale at which $\kappa_{e, 0}$ becomes zero. Model systems of lipid bilayers that are commonly used in laboratory studies do not exceed micrometer-range sizes; also, they typically have $\kappa\left(l_{0}\right) \sim 10 k_{B} T_{\text {room }}$, where $l_{0}$ $\simeq 10 \mathrm{~nm}$. A quick calculation based on Eq. (20) reveals that $\xi_{p}$ for these systems is far larger than their sizes. Hence the conformational stability of these systems is ensured in principle, as is often the case. However, we will argue with our results that such statements may no longer hold when inplane fields and their thermal fluctuations become relevant.

It is intuitively easy to anticipate that the effects of thermal fluctuations of in-plane fields, specifically, the difference field $\phi$, would be enhancing conformational fluctuations of the membrane surface. In fact, the mean-field theory already points towards such effects. For simplicity, we will only consider situations where the physical parameter $\mu$ is zero and the generalization to cases where $\mu$ is nonzero is fairly straightforward. The mean-field theory, which neglects the nonlinearity of the bending energy, then makes the following prediction for the height-height $(Z-Z)$ correlation function $G_{0}(\vec{q})$ in the high-temperature region where the in-plane fields are disordered:

$$
\frac{1}{G_{0}(\vec{q})}=\frac{\kappa q^{4}}{k_{B} T}\left[1-\frac{\lambda_{\mathrm{sc}}^{-2}}{\bar{\xi}_{\phi}^{-2}+\lambda_{\mathrm{sc}}^{-2}+q^{2}}\right],
$$

where $\bar{\xi}_{\phi}$, defined by $\bar{\xi}_{\phi}^{-2} \equiv t_{\Delta}-\lambda_{\text {sc }}^{-2}$, may be considered as an effective correlation length for the in-plane difference field. Hence $\bar{\xi}_{\phi}$ increases as the temperature is reduced. The effective bending rigidity $\kappa_{e}$, identified as the coefficient of the $q^{4}$ term in the small- $q$ expansion [40] of $1 / G_{0}(\vec{q})$, is then given by

$$
\kappa_{e}=\kappa\left(1-\frac{\lambda_{\mathrm{sc}}^{-2}}{\bar{\xi}_{\phi}^{-2}+\lambda_{\mathrm{sc}}^{-2}}\right) .
$$

The effect that the fluctuations in the $\phi$ field have in reducing the bending rigidity is made apparent by the mean-field correction to the bending rigidity. Furthermore, Eq. (22), which predicts the onset of strong surface fluctuations as $\bar{\xi}_{\phi}$ becomes divergingly large, itself signals the breakdown of the mean-field theory when the membrane is no longer subject to mechanical constraints.

Taking into consideration the nonlinearity of the bending energy, therefore, becomes necessary in dealing with the presence of strong surface fluctuations. To this end, we expand our model free energy (6) in terms of the power series in $Z(x, y)$ and include anharmonic terms up to the quartic power in $Z$ (while retaining only quadratic terms in the inplane difference field) and perform a simplified analysis of the free energy based on a field-theory approach [41]. In this analysis, the renormalization of the physical parameters other than $\kappa$ is neglected, an approximation that does not affect our principal conclusions (a more systematic analysis based on renormalization-group theories is presented elsewhere [42]). The present analysis yields a "renormalized" height-height correlation function $G(\vec{q})$ that contains nonlinear corrections calculated to one-loop order and the effective bending rigidity is again obtained as the coefficient of the $q^{4}$ term in the small- $q$ expansion of $[G(\vec{q})]^{-1}$. An inspection of the expanded elastic free energy [28] shows that only two Feynman (self-energy) diagrams contribute to the $q^{4}$ term of the renormalized $[G(\vec{q})]^{-1}$. These diagrams have the same topologies as those involved in the calculation that led to the renormalized bending rigidity given in Eq. (20) [27]; the only difference is in the expression for the linear (harmonic)order correlation function $G_{0}(\vec{q})$, which in our calculations is given by Eq. (21). Furthermore, the nonlinear (anharmonic) contribution arising from the bilinear coupling between $\phi$ and the mean curvature generates only vertices that make no contributions at the one-loop level. Summing over the two diagrams thus gives the renormalized $[G(\vec{q})]^{-1}$ (in the form of a Dyson equation)

$$
\frac{1}{G(\vec{q})}=\frac{1}{G_{0}(\vec{q})}-k_{B} T\left(1+\frac{1}{2}\right)\left[\int_{p_{0}}^{\Lambda} p^{2} G_{0}(\vec{p})\right] \kappa q^{4},
$$

where $p_{0}=1 / l$ and $\Lambda=1 / a_{0}$ represent the long- and shortwavelength cutoffs, respectively.

An expression for the effective bending rigidity follows from the evaluation of the integral in Eq. (23):

$$
\begin{aligned}
\bar{\kappa}_{e}(l)= & \kappa\left(a_{0}\right)\left(1-\frac{\lambda_{\mathrm{sc}}^{-2}}{\bar{\xi}_{\phi}^{-2}+\lambda_{\mathrm{sc}}^{-2}}\right) \\
& -\frac{3 k_{B} T}{4 \pi}\left[\ln (\Lambda l)+\frac{1}{2} \frac{\bar{\xi}_{\phi}^{2}}{\lambda_{\mathrm{sc}}^{2}} \ln \left(\frac{1+l^{2} \bar{\xi}_{\phi}^{-2}}{1+\Lambda^{-2} \bar{\xi}_{\phi}^{-2}}\right)\right] .
\end{aligned}
$$

The first term is the result from the harmonic approximation, which we have already encountered in Eq. (22). The second term arises from the one-loop correction and consists of two parts: The first is the same nonlinear contribution given in Eq. (19) and the second is a nontrivial nonlinear contribution from the bilinear coupling, which, to our knowledge, has not been reported and considered before.

From Eq. (24) an effective persistence length $\bar{\xi}_{p}$, "renormalized" by the fluctuations of the in-plane (difference) field, can be derived as a function of the effective in-plane correlation length $\bar{\xi}_{\phi}$ by setting $\bar{\kappa}_{e}(l)=0$ and replacing $l$ by $\bar{\xi}_{p}$ in the equation. $\bar{\xi}_{p}$ decreases as the in-plane correlation length increases at lowering the temperature. To illustrate semi-quantitatively the extent of the reduction in the membrane persistence length due to the in-plane fluctuations, we consider a particular situation where the persistence length 
coincides with the in-plane correlation length. The order of magnitude of the persistence length then depends on $\kappa\left(a_{0}\right)$ and $\lambda_{\mathrm{sc}}$. A numerical investigation shows that, with a reasonable estimate of $\lambda_{\mathrm{sc}}=\mathcal{O}(10) a_{0}$ and $\kappa\left(a_{0}\right)=10 k_{B} T$, the persistence length will be of the order $\bar{\xi}_{p}=\bar{\xi}_{\phi}=\mathcal{O}\left(10^{2}\right) a_{0}$. By comparing this with $a_{0} \exp \left[4 \pi \kappa\left(a_{0}\right) / 3 k_{B} T\right]$, the "bare" persistence length, it is easy to see that the reduction in the magnitude of the persistence length is rather striking. Furthermore, this remarkable extent of the reduction is very robust, not significantly influenced by the precise value of the cutoff.

\section{DISCUSSION}

We have presented a phenomenological model for fluid lipid bilayers, which focuses particularly on the interplay between monolayer cooperative phenomena and intermonolayer coupling within a bilayer. One basic aspect of intermonolayer coupling, which has its origin in both the bilayer architecture and the flexibility typical of a fluid bilayer, has been described in many previous studies of lipid bilayers in terms of a bilinear coupling between local transverse asymmetry (represented by the difference in the local orderparameter fields $\phi_{1}$ and $\phi_{2}$ of the two monolayers) and local mean curvature of the bilayer. However, we have included in our model another aspect of intermonolayer coupling, which arises from direct interactions between monolayers and have modeled it in terms of the simplest phenomenological form $\gamma_{12} \phi_{1} \phi_{2}$. Furthermore, we have modeled with a planar physical frame and an effective physical tension $\bar{\sigma}_{0}$ the effects of mechanical constraints that stabilize bilayer conformations.

Our study of the phenomenological model has largely been based on a mean-field analysis, in which neither the thermal fluctuations in the in-plane fields nor those in the bilayer conformation are explicitly dealt with. The main results of the analysis are summarized in Figs. 1-3 in terms of a series of phase diagrams illustrating both the equilibrium phases, a flat, disordered (D) phase, a flat, homogeneously ordered (HO1) phase with no bilayer transverse asymmetry, another flat, homogeneously ordered (HO2) phase, but with bilayer transverse asymmetry, and finally, phases of modulated (M1 and M2) structures and thermodynamic transitions between the different phases.

Similar types of mean-field calculations have been performed in other studies of phenomenological models of lipid bilayers and modulated phases have also been predicted $[9,12,14]$. Our analysis has not only been more systematic and extensive in exploring the effects of different phenomenological parameters, but more importantly has also revealed two new results. First, we have shown that, in different regions of the parameter space, modulated structures of different characteristics, specifically M1 and M2, can exist and changes from one structure to another can involve thermodynamic singularities (phase transitions). Second, we have demonstrated the nontrivial role of the direct intermonolayer interaction (as represented by $\gamma_{12}$ ), even when it is weak. Explicitly, we have shown that an attractive intermonolayer interaction $\left(\gamma_{12}<0\right)$ tends to reduce the region of stability of the modulated phases in the parameter space, while a repulsive interaction $\left(\gamma_{12}>0\right)$ always favors phases where the bilayer transverse symmetry is spontaneously broken, either locally (as in the modulated phases) or globally (as in the HO2 phase). Moreover, we have pointed out the effect of (even weak) $\gamma_{12}$ as a "symmetry-breaking" field, which in the low-temperature region of the parameter space selects the $\mathrm{HO} 1$ phases while attractive and selects the $\mathrm{HO} 2$ phases while repulsive.

The model we have presented and studied is only a phenomenological one and even at this level only a minimal one. We have no concrete knowledge on how to establish a specific, quantitative link between some of the model parameters and a given experimental system of lipid bilayers. For example, the parameter $\bar{C}_{0}$ has not been quantitatively and systematically analyzed and determined; we have introduced an effective tension $\bar{\sigma}_{0}$ to model the effects of mechanical constraints, while in experiments mechanical constraints are often imposed in the form of confinement (as in large multilamellar vesicles), a fixed area-volume ratio (as in the case of single large unilamellar vesicles), etc. Also the model, being phenomenological, inevitably misses some specific details of complicated interactions at work in an experimental system, as well as some other degrees of freedom present in a lipid bilayer, e.g., the molecular-tilt degrees of freedom. However, we still believe that the predictions of this model are useful as guidelines to systematically study and assess through experiments the complexity of the ordering phenomena in a fluid bilayer. First of all, a very crude estimate based on the order-of-magnitude values of some of the relevant physical parameters shows that the predicted domain sizes in the modulated phases [see Eq. (14)] fall into the range of length scales that are experimentally accessible or encountered. Typical values of the effective surface tension $\left(\bar{\sigma}_{0}\right)$ in giant vesicles have been measured [30], which span the range from $e^{-7}(0.001)$ to $e^{-1}(0.4) \mathrm{dyn} / \mathrm{cm}$. The often quoted value of the bending rigidity $\kappa$ is $5 \times 10^{-13} \mathrm{ergs}$ $\sim 10 k_{B} T_{\text {room }}$. If $c$ is chosen to be comparable to $k_{B} T_{\text {room }}$ and $\bar{C}_{0}^{-1}$ is taken to be in the range of tens of nanometers, then it is straightforward to see that the period of modulation is predicted to lie in the range of several hundreds to thousands of angstroms. Moreover, the phase diagrams we have presented constitute a generic picture of the complexity that might be encountered in experiments. As the lipid species composing a bilayer are varied (which a varying $\gamma_{12}$ may represent), as the molecular composition of a lipid mixture is changed (which may amount to changing $\mu$ ), or as the areavolume ratio is tuned (which may result in a change in $\bar{\sigma}_{0}$ ), individual sequences of equilibrium phases and thermodynamic transitions observed may resemble or differ from one another. At the same time, however, the phenomenological theory also indicates the possibility of "simple" mechanisms underlying complex phenomena.

Some of the characteristic features of the predicted phase behavior may have already been observed in certain experimental systems. For lipid bilayers of PC near their main (chain-melting) transitions, the relevance of the coupling between bilayer deformations and the monolayer density fields (or chain conformational states) has been supported by experiments [17]. Thus, in the presence of a nonzero surface tension or, equivalently, a nonzero osmotic pressure difference across a bilayer or a confinement potential, one might 
expect that the main phase transition is accompanied by an approximately sinusoidal modulation of the bilayer conformation as well as a periodical modulation of the density or thickness profile characteristic of the M2 structure, as the corresponding Landau-Ginzburg description involves a nonzero ordering field $\mu \propto T-T_{m}$ [see Eq. (5)]. Recent experiments [43] in fact show that upon cooling from the hightemperature fluid phase, $\mathrm{PC}$ lipid bilayers transform into a metastable "ripple phase"' $P_{\beta^{\prime}}(m)$, which is stable for hours [44]. This phase is characteristically different from the wellknown primary ripple phase, with a larger periodicity (around $270 \AA$, compared to a typical $130 \AA$ of the primary ripple), a symmetric profile of the ripple (contrasting the asymmetric profile of the primary ripple phase), and an appreciable lateral variation in the bilayer thickness [43]. This $P_{\beta^{\prime}}(m)$ may turn out to be the same as a so-called $\Lambda-P_{\beta^{\prime}}$ phase observed in earlier freeze-fracture studies [45]. The bilayer structure in the $\Lambda-P_{\beta^{\prime}}$ phase also has a symmetric profile of modulation and sometimes characteristic grooves are found on the ridges of the ripples. Analysis of the lateral defects suggests that there is no acyl-chain tilt with respect to the overall bilayer plane [46]. These properties would be the characteristics expected of the predicted M2 structure of our model should the thickness of each monolayer be thought to depend on the corresponding in-plane field $\phi_{i}$ [5]. Moreover, upon further cooling, the $P_{\beta^{\prime}}(m)$ phase becomes unstable with respect to a nonmodulated $L_{\beta^{\prime}}$ phase [43]. This sequence of phase transitions and the characteristic phases involved overall seem to be qualitatively consistent with the predicted cooling scenarios that involve modulated structures (see the phase diagrams in Fig. 3). This rather plausible interpretation of the $P_{\beta^{\prime}}(\mathrm{m})$ would imply that the ripple phase involves alternating domains where lipid chains appear either ordered or disordered. In fact, this idea has been advocated by several other studies $[47,48]$ and is supported by the experimental finding that more than $20 \%$ of the lipids in this particular ripple phase appear in a state more characteristic of the fluid phase of lipid bilayers [49].

Another class of lipid systems that display phenomena of length-scale selection are systems of lipid mixtures. Bilayer surface modulations of very long lengths with accompanying compositional variation have been found [50]. For example, in PC-cholesterol mixtures that show coexistence of the gel and the liquid-ordered phases [5] domains have been observed by using scattering techniques to have sizes that range from 40 to $60 \AA$ and that depend on both the temperature and the overall composition of the mixtures [51,52]. It has also been found that the domain size and the associated (scattering) intensity actually grow as the temperature is decreased. This trend is also predicted for the M2 phase by our model calculations.

We note, however, that the mean-field analysis discussed so far is based on the specific model (2), which presents perhaps the simplest phenomenological description of a fluid lipid bilayer and therefore inevitably implies certain simplification of some physical effects. One such effect, neglected in our model, is the dependence of the bending rigidity of a monolayer on the corresponding in-plane field. Theoretical studies of lipid bilayers of mixtures [53] have predicted a linear dependence, i.e., $\kappa_{m}\left(\phi_{i}\right) \approx \kappa_{0}+\alpha_{\kappa} \phi_{i}$, and recent experiments [54] have started to explore this issue. Taking this linear dependence into account would give rise to terms such as $\psi H^{2}$ and $\phi \psi H$, which could also be written down based on considerations of the inversion symmetry of the bilayer. It would be expected that the presence of these terms in the bilayer free energy leads to certain modifications of our mean-field predictions. However, it may be argued that these terms do not affect the existence of the D-M1 and M1-M2 transitions, although they must lead to quantitative changes both in the precise locations of these transitions in the parameter space and in the wavelength of modulation of the modulated phases. It may also be readily seen that these terms will not influence the transitions between those phases in which the bilayer remains flat.

Of course, thermal fluctuations, which have been neglected in the mean-field analysis, may also modify the mean-field predictions in various aspects. The modulated phases in our analysis are considered to be striped. Based on an argument formulated by Toner and Nelson, however, it may be anticipated that thermal fluctuations eliminate any long-range correlations between the orientations of the stripes. In other words, topological defects may be expected to decorate the modulated (striped) phases [55]. The region of stability of the modulated phases in the mean-field phase diagrams may also be modified by thermal fluctuations. It has been established that in systems involving mechanisms of length-scale selection thermal fluctuations can destabilize a homogeneous, disordered phase towards a modulated phase and furthermore change the nature of the transition between the two phases from second order to first order [56]. Thus, in the presence of thermal fluctuations, the D-M phase boundaries may appear in different locations in the parameter space from those shown in the mean-field phase diagrams and may be of first order rather than second order.

In Sec. IV we have extended our analysis of the phenomenological model (2) beyond the scope of the mean-field theory to investigate some of the macroscopic effects of the intermonolayer coupling in situations where mechanical constraints are no longer enforced and consequently strong fluctuations in the bilayer conformation are expected. Qualitatively stated, our main conclusion is that strong fluctuations in the in-plane fields, through the bilinear coupling between bilayer local mean curvature and the in-plane difference field, enhance bilayer conformational fluctuations and this interplay leads to a dramatic reduction of the effective bending rigidity. This result implies that in the absence of external mechanical constraints, a fluid lipid bilayer may lose the correlation between its surface normals beyond a persistence length $\bar{\xi}_{p}[38,57]$ that can be reduced by "turning on', strong fluctuations in the in-plane fields. As our estimate made in Sec. IV shows, this effective persistence length may be reduced to a range that is likely accessible to laboratory experiments. In other words, the "crumpling instability,", or the conformational collapse, of a bilayer may be observed at realistic length scales (see Sec. IV). Moreover, this mechanism may provide a minimal explanation for the dramatic influence that cosurfactants can have on the structural stability of amphiphilic multimembrane systems [38,57].

Finally, it is worth pointing out that the bilayer softening effect of in-plane fluctuations is also present in bilayers that are under mechanical constraints, although it does not imply conformational instability of the bilayers. This effect has ac- 
tually been used to interpret the phenomenon of "anomalous swelling" observed in multilamellar systems of onecomponent PC lipids when the systems are cooled towards their main phase transition points $[17,58]$. The measurement of an effective or " apparent" bending rigidity by an analysis of flicker noise (bilayer shape fluctuations) of individual vesicles has also demonstrated this effect [59].

\section{ACKNOWLEDGMENTS}

We have benefited greatly from discussions with $\mathrm{T}$. Hønger, M. Sabra, T. Gil, and O. G. Mouritsen. This work was supported by the Danish Natural Science Research Council and the Technical University of Denmark.

\section{APPENDIX}

This appendix collects the available analytical expressions for some of the lines of phase transitions and special points in the phase diagrams shown in Figs. 1 and 3. In Fig. 1 the line of the second-order transitions between the D and HO1 phases is simply given by

$$
t_{\Delta}=-\gamma_{12} \text {. }
$$

The critical line separating the D and the M1 phases is described by

$$
t_{\Delta}=\alpha\left(q_{0}\right)
$$

where $q_{0}$ is the wave number of modulation whose definition is given in Eq. (14)

$$
\alpha\left(q_{0}\right)=\xi_{c}^{-2}\left(\sqrt{\frac{\xi_{c}^{2}}{\lambda_{\mathrm{sc}}^{2}}-1}\right)^{2} .
$$

The line of first-order transitions between the HO1 and the M1 phases is expressed as

$$
t_{\Delta}=\frac{-\sqrt{\frac{3}{2}} \gamma_{12}-\alpha\left(q_{0}\right)}{\sqrt{\frac{3}{2}}-1} .
$$

The regions of the M1 and the M2 structures are separated by a line of second-order transtions, given by

$$
t_{\Delta}=-\left(2 q_{0}^{2}+\gamma_{12}\right)-\sqrt{2\left(\alpha\left(q_{0}\right)+2 q_{0}^{2}+\gamma_{12}\right)^{2}-4 q_{0}^{4}} .
$$

Finally, the first-order transitions between the M1 and the HO2 phases fall onto a straight line, described by

$$
t_{\Delta}=\frac{-\alpha\left(q_{0}\right)}{\sqrt{\frac{3}{2}}-1}
$$

Some of the special points denoted in Fig. 1 are determined as follows: $P_{L}$ is the point of intersection of the transition line given by Eq. (A1) and the line described by Eqs. (A3) and (A4). The two critical end points, $P_{\mathrm{ce}, 1}$ and $P_{\mathrm{ce}, 2}$ are the points of intersection of the critical M1-M2 line [Eq. (A5)] with the first-order M1-HO1 line [Eq. (A4)] and the first-order $\mathrm{M} 1-\mathrm{HO} 2$ [Eq. (A6)], respectively. For a fixed $\lambda_{\mathrm{sc}}, \xi_{c, M}^{2}$ defines the value of $\xi_{c}^{2}$ at which $P_{c e, 1}$ and $P_{\mathrm{ce}, 2}$ merge.

In Fig. 3 the critical surfaces separating the hightemperature (D) phase from the modulated (M) phase are given by

$$
\begin{gathered}
\left(t_{\Delta}+\gamma_{12}\right) \psi^{(0)}+\frac{1}{6}\left(\psi^{(0)}\right)^{3}=\mu, \\
t_{\Delta}=\alpha\left(q_{0}\right)-\frac{1}{2}\left(\psi^{(0)}\right)^{2} .
\end{gathered}
$$

The coordinates of the two tricritical points $P_{\mathrm{tc}, 1}$ and $P_{\mathrm{tc}, 2}$ are obtained by substituting into Eq. (A7) the following two expressions for $\psi^{(0)}$, respectively:

$$
\psi^{(0)}= \pm \sqrt{\frac{\left[\gamma_{12}+\alpha\left(q_{0}\right)+4 q_{0}^{2}\right]\left[\gamma_{12}+\alpha\left(q_{0}\right)\right]}{2\left[\gamma_{12}+\alpha\left(q_{0}\right)+4 q_{0}^{2}\right]+\left[\gamma_{12}+\alpha\left(q_{0}\right)\right]}}
$$

[1] For a comprehensive review of physics of biomembranes refer to Structure and Dynamics of Membranes: From Cells to Vesicles; Generic and Specific Interactions, Vol. 1A-B of Handbook of Biological Physics, edited by R. Lipowsky and E. Sackmann (North-Holland, Amsterdam, 1995).

[2] P. B. Canham, J. Theor. Biol. 26, 61 (1970).

[3] W. Helfrich, Z. Naturforsch. C 28, 693 (1973).

[4] Statistical Mechanics of Membranes and Interfaces, edited by D. Nelson, T. Piran, and S. Weinberg (World Scientific, Singapore, 1989).

[5] M. Bloom, E. Evans, and O. G. Mouritsen, Q. Rev. Biophys. 24, 293 (1991).

[6] W. Knoll, G. Schmidt, K. Ibel, and E. Sackmann, J. Chem. Phys. 79, 3439 (1983).

[7] J. H. Ipsen, G. Karlström, O. G. Mouritsen, H. Wennerström, and M. J. Zuckermann, Biochim. Biophys. Acta 905, 162 (1987).

[8] S. Leibler, J. Phys. (Paris) 47, 507 (1986).

[9] S. Leibler and D. Andelman, J. Phys. (Paris) 48, 2013 (1987).

[10] S. A. Safran, P. Pincus, and D. Andelman, Science 248, 354 (1990); S. A. Safran, P. Pincus, D. Andelman, and F. C. MacKintosh, Phys. Rev. A 43, 1071 (1991).

[11] F. C. MacKintosh and S. A. Safran, Phys. Rev. E 47, 1180 (1993).

[12] F. C. MacKintosh, Phys. Rev. E 50, 2891 (1994).

[13] D. Andelman, T. Kawakatsu, and K. Kawasaki, Europhys. Lett. 19, 57 (1992); T. Kawakatsu, D. Andelman, K. Kawasaki, and T. Taniguchi, J. Phys. II 3, 971 (1993); T. Taniguchi, K. Kawasaki, D. Andelman, and K. Kawakatsu, ibid. 4, 1333 (1994). 
[14] H. Kodama and S. Komura, J. Phys. II 3, 1305 (1993).

[15] U. Seifert, Phys. Rev. Lett. 70, 1335 (1993).

[16] M. M. Kozlov and W. Helfrich, Langmuir 8, 2792 (1992); W. Helfrich and M. M. Kozlov, J. Phys. II 3, 287 (1993).

[17] T. Hønger, K. Mortensen, J. H. Ipsen, J. Lemmich, R. Bauer, and O. G. Mouritsen, Phys. Rev. Lett. 72, 3911 (1994).

[18] B. Durhuus, J. Frölich, and T. Jonsson, Nucl. Phys. B 225, 185 (1983).

[19] L. D. Landau and E. M. Lifshitz, Statistical Physics Part 1, Vol. 5 in Course of Theoretical Physics, 3rd ed. (Pergamon, New York, 1980).

[20] The numerical prefactor $1 / 2$ in Eq. (3) is chosen merely for the convenience of normalization regarding Eq. (6).

[21] K. Jørgensen, J. H. Ipsen, O. G. Mouritsen, D. Bennett, and M. J. Zuckermann, Biochim. Biophys. Acta 1062, 227 (1991); W. Knoll, G. Schmidt, H. Rötzer, T. Henkel, W. Pfeiffer, E. Sackmann, S. Mittler-Neher, and J. Spinke, Chem. Phys. Lipids 57, 363 (1991); M. Luxnat and H.-J. Galla, Biochim. Biophys. Acta 856, 274 (1986); D. Barthel, O. Zschoernig, K. Lange, R. Lenk, and K. Arnold, ibid. 945, 361 (1988).

[22] S. Doniach, J. Chem. Phys. 68, 4912 (1978).

[23] U. Seifert and S. A. Langer, Europhys. Lett. 23, 71 (1993).

[24] D. Andelman, in Structure and Dynamics of Membranes: From Cells to Vesicles; Generic and Specific Interactions (Ref. [1]), Chap. 12; M. Kiometzis and H. Kleinert, Phys. Lett. A 140, 520 (1989); B. Duplantier, R. E. Goldstein, V. RomeroRochin, and A. I. Pesci, Phys. Rev. Lett. 65, 508 (1990).

[25] N. Düzgünes, C. Newton, K. Fisher, J. Fedor, and D. Papahadjopoulos, Biochim. Biophys. Acta 944, 391 (1988).

[26] F. David and S. Leibler, J. Phys. II 1, 959 (1991).

[27] L. Peliti and S. Leibler, Phys. Rev. Lett. 54, 1690 (1985).

[28] H. Kleinert, Phys. Lett. 114A, 263 (1986).

[29] W. Helfrich, J. Phys. (Paris) 46, 1263 (1985).

[30] E. Evans and W. Rawicz, Phys. Rev. Lett. 64, 2094 (1990).

[31] W. Cai, T. C. Lubensky, P. Nelson, and T. Powers, J. Phys. II 4, 931 (1994).

[32] J. L. Harden and F. C. MacKintosh, Europhys. Lett. 28, 495 (1994).

[33] In fact, $\left\{I, \mathcal{O}_{1}, \mathcal{O}_{2}, \mathcal{O}_{3}\right\}$, where $I$ is a unity operator, form an Abelian group.

[34] In this paper, the phase diagrams are given when the two control parameters are chosen to be either $\left(t_{\Delta}, \gamma_{12}\right)$ or $\left(t_{\Delta}, \mu\right)$. However, the phase diagrams have also been calculated in the parameter space spanned by $\left(t_{\Delta}, \bar{\sigma}_{0}\right)$, for $\mu=0$ and different (fixed) values of $\gamma_{12}$; those phase diagrams have been presented in Ref. [35].

[35] L. Miao, P. L. Hansen, and J. H. Ipsen, in Dynamical Networks in Physics and Biology, edited by. D. Beysens and G. Forgać (Springer, New York, in press).
[36] It follows from Eqs. (12)-(14) that the two effective parameters resulted from $\bar{\sigma}_{0}, \kappa$, and $\bar{C}_{0}$ are $\xi_{c}^{-2}$ and $\lambda_{\mathrm{sc}}^{-2}$.

[37] It is likely that more types of modulated structures would appear as equilibrium states, if the ansatz is made more sophisticated to include modes of higher multiples of the wave number $\bar{q}_{0}$.

[38] P. G. de Gennes and C. Taupin, J. Phys. Chem. 86, 2294 (1982).

[39] J. H. Ipsen and C. Jeppesen, J. Phys. I 5, 1563 (1995).

[40] The small- $q$ expansion implies that $q \ll \lambda_{\mathrm{sc}}^{-1}$.

[41] D. J. Amit, Field Theory, the Renormalization Group, and Critical Phenomena (World Scientific, Singapore, 1984).

[42] P. L. Hansen, Ph.D. thesis, The Technical University of Denmark, 1997 (unpublished).

[43] M. Rappolt and G. Rapp, Eur. Biophys. J. 24, 381 (1996).

[44] B. Tenchov, Chem. Phys. Lipids 57, 165 (1991).

[45] C. Gebhardt, H. Gruler, and E. Sackmann, Z. Naturforsch. C 32, 581 (1977).

[46] E. Sackmann, D. Rueppel, and C. Gebhardt, in Liquid Crystals of One- and Two-Dimensional Order, edited by W. Helfrich and W. Heppke (Springer, Berlin, 1980).

[47] K. Tsuchida and I. Hatta, Biochim. Biophys. Acta 945, 73 (1988).

[48] K. Honda and H. Kimura, J. Phys. Soc. Jpn. 60, 1212 (1991).

[49] K. Tsuchida, Y. Tamura, and I. Hatta, J. Phys. Soc. Jpn. 67, 4151 (1993).

[50] E. Sackmann, in Physical Basis of Self-Organization and Function of Membranes: Physics of Vesicles, Vol. 1A of Structure and Dynamics of Membranes: From Cells to Vesicles; Generic and Specific Interactions (Ref. [1]).

[51] A. Hicks, M. Dinda, and M. A. Singer, Biochim. Biophys. Acta 903, 177 (1987).

[52] K. Mortensen, W. Pfeiffer, E. Sackmann, and W. Knoll, Biochim. Biophys. Acta 945, 221 (1988).

[53] S. May and A. Ben-Shaul, J. Chem. Phys. 103, 3839 (1995); R. Netz and P. Pincus, Phys. Rev. E 52, 4114 (1995).

[54] S. Leikin, M. M. Kozlov, N. L. Fuller, and R. P. Rand, Biophys. J. 71, 2623 (1996).

[55] J. Toner and D. Nelson, Phys. Rev. B 23, 316 (1981).

[56] S. A. Brazovskii, Zh. Éksp. Teor. Fiz. 68, 175 (1975) [Sov. Phys. JETP 41, 85 (1975)].

[57] D. Roux, C. Coulon, and M. E. Cates, J. Phys. Chem. 96, 4174 (1992), and references therein.

[58] F. Y. Chen, W. C. Hung, and H. W. Huang, Phys. Rev. Lett. 79, 4026 (1997).

[59] L. Fernandez-Punte, I. Bivas, M. D. Mitov, and P. Méléard, Europhys. Lett. 28, 181 (1994). 